\title{
152. Note on Free Algebraic Systems
}

\author{
By Tsuyoshi FujIwara \\ Department of Mathematics, Yamaguchi University \\ (Comm. by K. ShODA, M.J.A., Nov. 12, 1956)
}

In his paper, ${ }^{1)} \mathrm{K}$. Shoda has defined only the free $A$-algebraic systems, when he has discussed the free algebraic systems. However, in this note, we shall define free algebraic systems more generally. And we shall show a generalization of the Shoda's fundamental theorem $^{2)}$ (Theorems 1, 2 and 3), and a necessary and sufficient condition for the existence of the free algebraic system with an arbitrary set of relations (Theorem 3). Finally, we shall show a characterization of the algebraic systems defined by only a set of relations, i.e. the $A$-algebraic systems satisfying a set of relations (Theorem 4).

Throughout this note, the system $V$ of single-valued compositions will be fixed. Let $E$ be a set of generators, then the absolutely free algebraic system $F(E, \phi)^{3)}$ is defined. And let $P$ be a family of postulates with respect to $V$ and $E$, then $P$-algebraic systems generated by $E$ are defined as residue class systems of $F(E, \phi)$ satisfying $P$. And $(E, P)$ denotes the set of all $P$-algebraic systems generated by $E$. Moreover, let $R$ be a set of relations (identities) in $F(E, \phi)$, then the $P$-algebraic systems satisfying $R$ generated by $E$ are defined. And $(E, P, R)$ denotes the set of all such $P$-algebraic systems.

An algebraic system $\mathfrak{F}$ is called a free $P$-algebraic system with a set $R$ generated by $E$, or a free algebraic system belonging to $(E, P, R)$, when $\widetilde{\mho}$ is contained in $(E, P, R)$ and every algebraic system in $(E, P, R)$ is a residue class system of $\widetilde{\mho}$. And we denote it by $F(E, P, R)$.

Theorem 1. If an algebraic system $\mathfrak{A}$ is contained in $(E, P, R)$, then there exists a set $S$ of relations satisfying $\mathfrak{U}=F(E, P, S)$ and $S \supseteq R$.

Proof. Let $\mathfrak{A} \in(E, P, R)$, then it is clear that $\mathfrak{A} \in(E, \phi, R) .^{4)}$ Hence there exists a set $S$ of relations satisfying $\mathfrak{A}=F(E, \phi, S)$ and $S \supseteq R$ by

1) K. Shoda: Allgemeine Algebra, Osaka Math. J., 1 (1949).

2) Using our notations, we can show the Shoda's fundamental theorem for the free algebraic systems as follows: Let $P$ be a family of composition-identities. Then i) there exists a free algebraic system $F(E, P, R)$ for every set $R$ of relations, ii) if an algebraic system $\mathfrak{A}$ is contained in $(E, P, R)$, then there exists a set $S$ of relations satisfying $\mathfrak{A}=F(E, P, S)$ and $S \supseteq R$, and iii) if $R \subseteq S$, then $F(E, P, S)$ is a residue class system of $F(E, P, R)$.

3) In his paper 1$)$, K. Shoda has denoted by $O(E)$ the absolutely free algebraic system.

4) $\phi$ denotes the empty set. 
the Shoda's fundamental theorem. Now, it is verified that $\mathfrak{A} \in(E, P, S)$, because $\mathfrak{U} \in(E, P, R)$ and $\mathfrak{U} \in(E, \phi, S)$. Moreover, any algebraic system in $(E, P, S)$ is a residue class system of $\mathfrak{A}$, since $\mathfrak{A}=F(E, \phi, S) \in(E, P, S)$ $\subseteq(E, \phi, S)$. Hence $\mathfrak{I}$ is a free algebraic system belonging to $(E, P, S)$, i.e. $\mathfrak{A}=F(E, P, S)$.

Theorem 2. Suppose that there exists $F(E, P, R)$ for every set $R$ of relations. If $R \subseteq S$, then $F(E, P, S)$ is a residue class system of $F(E, P, R)$.

Proof. Let $R \subseteq S$, then it is clear that $(E, P, S) \subseteq(E, P, R)$. Hence $F(E, P, S) \in(E, P, R)$. Therefore $F(E, P, S)$ is a residue class system of $F(E, P, R)$.

Let $R$ and $S$ be two sets of relations in $F(E, \phi)$. A condition that $R$ implies $S$ is called an implication from $R$ to $S$, or simply an implication. Moreover, a family $P$ of postulates is said to be equivalent to $P^{*}$, when $(E, P)=\left(E, P^{*}\right)$.

Theorem 3. In order that there exists $F(E, P, R)$ for every set $R$ of relations, it is necessary and sufficient that $P$ is equivalent to a family $P^{*}$ of implications.

(I) Proof of sufficiency: Let $P$ be equivalent to a family $P^{*}$ of implications, then it is obvious that $(E, P, R)=\left(E, P^{*}, R\right)$ for every set $R$ of relations. Hence it is sufficient to prove the existence of $F\left(E, P^{*}, R\right)$ for any set $R$ of relations.

Let $\mathfrak{A}$ be any algebraic system contained in $\left(E, P^{*}, R\right)$. It is, of course, clear that $\mathfrak{A} \in(E, \phi)$. Hence there exists a congruence $\theta_{\mathfrak{I}}$ of $F(E, \phi)$ satisfying $\mathfrak{A}=F(E, \phi) / \theta_{\mathfrak{Q}} .{ }^{5)} \quad$ Now, let $\varphi_{R}=\bigcap_{\mathfrak{A} \in\left(E, P^{*}, R\right)} \theta_{\mathfrak{A}}$, then it is verified that $F(E, \phi) / \varphi_{R} \in\left(E, P^{*}, R\right)$, since $P^{*}$ is a family of implications. And it is clear that any algebraic system $\mathfrak{A}$ in $\left(E, P^{*}, R\right)$ is a residue class system of $F(E, \phi) / \varphi_{R}$, since $\mathfrak{A}=F(E, \phi) / \theta_{\mathfrak{Y}}$ and $\theta_{\mathfrak{U}} \geqq \varphi_{R}$. Hence $F(E, \phi) / \varphi_{R}$ is a free algebraic system belonging to $\left(E, P^{*}, R\right)$, i.e. there exists a free algebraic system $F\left(E, P^{*}, R\right)$ $=F(E, \phi) / \varphi_{R}$.

(II) Proof of necessity: Suppose that there exists $F(E, P, R)$ for every set $R$ of relations. First we shall define a set $\bar{R}$, for every set $R$, as the set-sum of all sets $S$ satisfying $F(E, P, R)=F(E, P, S)$. Then it is verified that

$$
F(E, P, R)=F(E, P, \bar{R})=F(E, \phi, \bar{R})
$$

for every set $R$ of relations. Now we shall define $P^{*}$ as the family of all the implications written in the form that $R$ implies $\bar{R}$. In the following, we shall show the fact that $P$ is equivalent to $P^{*}$, i.e. $(E, P)=\left(E, P^{*}\right)$.

Let $\mathfrak{U} \in(E, P)$, then there exists a set $R$ of relations satisfying

5) $F(E, \phi) / \theta_{\mathfrak{Q}}$ denotes the residue class system of $F(E, \phi)$ modulo $\theta_{\mathfrak{Q}}$. 
$\mathfrak{U}=F(E, P, R)$ by Theorem 1 . And by $(*)$ we get that $\mathfrak{A}=(E, P, R)$ $=F(E, \phi, \bar{R})$. Now it is verified that $S \subseteq \bar{R}$ implies $\bar{S} \subseteq \bar{R}$ by Theorem 2. Hence $F(E, \phi, \bar{R})$ satisfies the family $P^{*}$ of implications. Therefore, $(E, P) \subseteq\left(E, P^{*}\right)$.

Hereafter we shall prove that $(E, P) \supseteq\left(E, P^{*}\right)$. It has been verified in (I) that there exists $F\left(E, P^{*}, R\right)$ for every set $R$ of relations. Hence we can define $R$, for every set $R$, as the set-sum of all sets $S$ satisfying $F\left(E, P^{*}, R\right)=F\left(E, P^{*}, S\right)$. Now let $\mathfrak{U} \in\left(E, P^{*}\right)$, then there exists a set $R$ of relations satisfying $\mathfrak{A}=F\left(E, P^{*}, R\right)$. And it is clear that $\mathfrak{A}=F\left(E, P^{*}, R\right)=F(E, \phi, \widetilde{R})$. Now it is evident that $\widetilde{R} \subseteq \overline{\widetilde{R}}$. And it is verified that $\widetilde{R} \supseteq \overline{\widetilde{R}}$, since $\mathfrak{A}$ satisfies the family $P^{*}$ containing the implication from $R$ to $\overline{\widetilde{R}}$. Hence $\widetilde{R}=\overline{\widetilde{R}}$ and $\mathfrak{A}=F(E, \phi, R)$ $=F(E, \phi, \overline{\widetilde{R}})$. Moreover, $F(E, \phi, \overline{\widetilde{R}})=F(E, P, \overline{\widetilde{R}})$ by $(*)$. Therefore, we get that $\mathfrak{A} \in(E, P),\left(E, P^{*}\right) \subseteq(E, P)$, and hence $(E, P)=\left(E, P^{*}\right)$.

Theorem 4. In order that (i) there exists $F(E, P, R)$ for every set $R$ of relations, and (ii) any residue class system of an algebraic system in $(E, P)$ is contained in $(E, P)$, it is necessary and sufficient that $P$ is equivalent to a family $P^{*}$ of relations.

Proof. The sufficient part of this theorem is evident. Hereafter we shall prove the necessary part. Suppose that $P$ satisfies the conditions (i) and (ii). Then there exists $F(E, P, \phi)$. And $F(E, P, \phi) \epsilon$ $(E, P) \subseteq(E, \phi)$. Hence there exists a set $P^{*}$ of relations satisfying $F\left(E, \phi, P^{*}\right)=F(E, P, \phi)$, i.e. $F\left(E, P^{*}, \phi\right)=F(E, P, \phi)$. Now we shall show that $P$ is equivalent to $P^{*}$, i.e. $(E, P)=\left(E, P^{*}\right)$. Let $\mathfrak{A} \in\left(E, P^{*}\right)$, then there exists a congruence $\theta$ of $F\left(E, P^{*}, \phi\right)$ satisfying $\mathfrak{U}=F\left(E, P^{*}, \phi\right) / \theta$. And clearly $\mathfrak{A}=F(E, P, \phi) / \theta$. Hence $\mathfrak{A} \in(E, P)$, and $\left(E, P^{*}\right) \subseteq(E, P)$. The converse $(E, P) \subseteq\left(E, P^{*}\right)$ is similarly obtained as mentioned above. Hence $(E, P)=\left(E, P^{*}\right)$. 